\title{
A prospective case series on surgical treatment of circumferential and semi-circumferential defects due to peri-implantitis
}

\author{
Luigi CANULLO(a) ii \\ Luca SIGNORINI(b) \\ Roberto PISTILLI(b) \\ Romeo PATINI(c) \\ Valeria PISTILLI(b) (iD \\ Paolo PESCE(d) ID \\ (a) Independent researcher, Rome, Italy. \\ (b) Private Practice, Rome, Italy. \\ (c) Catholic University of Sacred Heart, \\ Research Fellow, Rome, Italy. \\ (d) University of Genoa, Department of Surgical \\ Sciences (DISC), Implant and Prosthetic \\ Dentistry Unit, Ospedale S. Martino \\ Genoa, Italy.
}

Declaration of Interests: The authors certify that they have no commercial or associative interest that represents a conflict of interest in connection with the manuscript.

\section{Corresponding Author:}

Luigi Canullo

E-mail: lvigicanullo@yahoo.com

https://doi.org/10.1590/1807-3107bor- 2019.vol33.0072

\begin{abstract}
Different surgical treatment strategies for peri-implantitis with the use of graft material and membranes have been suggested without any longitudinal remarkable success rate. The present preliminary study was aimed to analyze a new clinical approach based on the disinfection of the implant connection, the disinfection of the implant surface and GBR approach in the treatment of circumferential and semi-circumferential bony defect resulting from peri-implantitis. Six consecutive patients were selected for the present study. After removal of factors that could potentially influence peri-implant pathology, the prosthetic rehabilitation was always removed and a full thickness flap was elevated to allow access to the peri-implant defect and the exposed implant surface. Once the defect was degranulated and the implant surface cleaned, a mixture (50:50) of autogenous bone and allograft was used. Guided bone regeneration technique using membranes was adopted and the flaps were closed for a submerged healing. Six months thereafter, a new re-opening procedure was performed and cleaned superstructures and crowns were repositioned. Patients were followed for one year thereafter and recalled for a customized oral hygiene every three months. Radiological and periodontal analysis was performed before surgery and every six months. The studied procedure was associated with a pronounced increase in REC and CAL with stable peri-implant conditions at 6 and 12 months. PI, BOP and PD values were significantly reduced both at 6 and 12 months. At 12 months, a mean PD gain of $4.5 \mathrm{~mm}$ and a bone loss reduction of $5.1 \mathrm{~mm}$ was obtained. Within the limitation of the present preliminary study, the proposed technique might represent a promising result for treatment of circumferential and semi-circumferential bone defects around implants affected by peri-implantitis.
\end{abstract}

Keywords: Peri-Implantitis; Decontamination.

\section{Introduction}

Nowadays the pathological conditions termed "mucositis" and Accepted for publication: June 13, 2019 Last revision: June 19, 2019 "peri-implantitis" are considered the major complication in dental implantology. ${ }^{1}$ 
A recent review reported that the prevalence of peri-implant mucositis and peri-implantitis ranged from 19 to $65 \%$ and from 1 to $47 \%$, respectively. Additionally, the meta-analyses revealed a weighted mean prevalence of peri-implant mucositis and peri-implantitis of $43 \%$ and $22 \%$, respectively. ${ }^{2}$

However, case definitions varied among the scientific literature. ${ }^{2}$ As a consequence, implant defined as affected by some authors might not be considered affected by other authors if another definition is used. Consequently, the prevalence could broadly vary between studies. ${ }^{3}$

Similarly, the therapy of peri-implantitis has been widely investigated by the scientific literature proposing different solutions including nonsurgical, surgical, resective, regenerative and combined approaches, all of them are aiming to stop the disease progression and bone loss by removing the bacterial infection and controlling the tissue inflammation.

Furthermore, surgical treatment includes the decontamination of the implant surface and the debridement of the bone defect. Various surface decontamination methods have been proposed to surgically treat periimplantitis such as the use of citric acid, delmopinol, chlorhexidine irrigation, air-powder abrasive unit, rotating brush with pumice, carbon dioxide laser, or gauze/cotton pellets soaked in saline and/or chlorhexidine. ${ }^{4}$ However, there is still a lack of evidence concerning their indication and outcome.

There have been numerous case reports, case series and clinical trials reporting on Guided Bone Regeneration (GBR) techniques in peri-implantitis treatment, in which a combination of both membrane and bone graft substitutes was used. ${ }^{5}$ However, there is limited evidence of success and reliability of that treatment protocol. ${ }^{5}$

The present preliminary study was aimed to analyze a new clinical approach based on the disinfection of the implant connection, disinfection of the implant surface and GBR approach in the treatment of circumferential and semi-circumferential bony defect resulting from peri-implantitis.

\section{Methodology}

In January 2016, in three different clinical centers in Rome, a preliminary prospective clinical trial was designed to test the efficacy of a GBR protocol in the treatment of bony defect resulting from peri-implantitis. Only patients having a periapical baseline radiograph obtained after prosthetic rehabilitation, older than 18 years and presenting a circumferential or semi-circumferential peri-implant bony defects were included in the study. ${ }^{6}$ Exclusion criteria were: relevant medical conditions (ASA III and IV); pregnant and lactating patients; patients with a history of bisphosphonate therapy (Figure 1).

Patients were informed about the study and the intervention, and were asked to sign an informed consent document in order to be involved. The investigation was conducted according to the principles embodied in the Helsinki Declaration.

\section{Pre-surgical phase}

Factors possibly influencing peri-implant pathology were evaluated and problems solved before the surgical phase. This included: occlusal adjustment, modification or change of badly designed prosthetic rehabilitations and periodontal treatment when necessary. Two weeks prior to the surgical therapy all patients received professional prophylaxis (full oral disinfection) and Chlorhexidine digluconate $0.12 \%$ (rinses twice a day, GUM Paroex 0.12\%, Sunstar Suisse, Etoy, Switzerland) was prescribed. Antibiotic treatment consisting of Amoxiciline $850 \mathrm{mg}$ + clavulanate $125 \mathrm{mg}$ every 8 hours was prescribed to all patients starting 2 days before the surgical treatment and finalizing 1 week after the intervention.

\section{Surgical phase}

The surgical intervention was performed under local anesthesia with articaine $4 \%$ and epinephrine 1:100.000 (Laboratorios Inibsa, Barcelona, Spain). The prosthetic rehabilitation was always removed and a full thickness flap was elevated to allow access to the peri-implant defect and the exposed implant surface.

Once granulation tissue was carefully removed, the implant surface was mechanically debrided and 


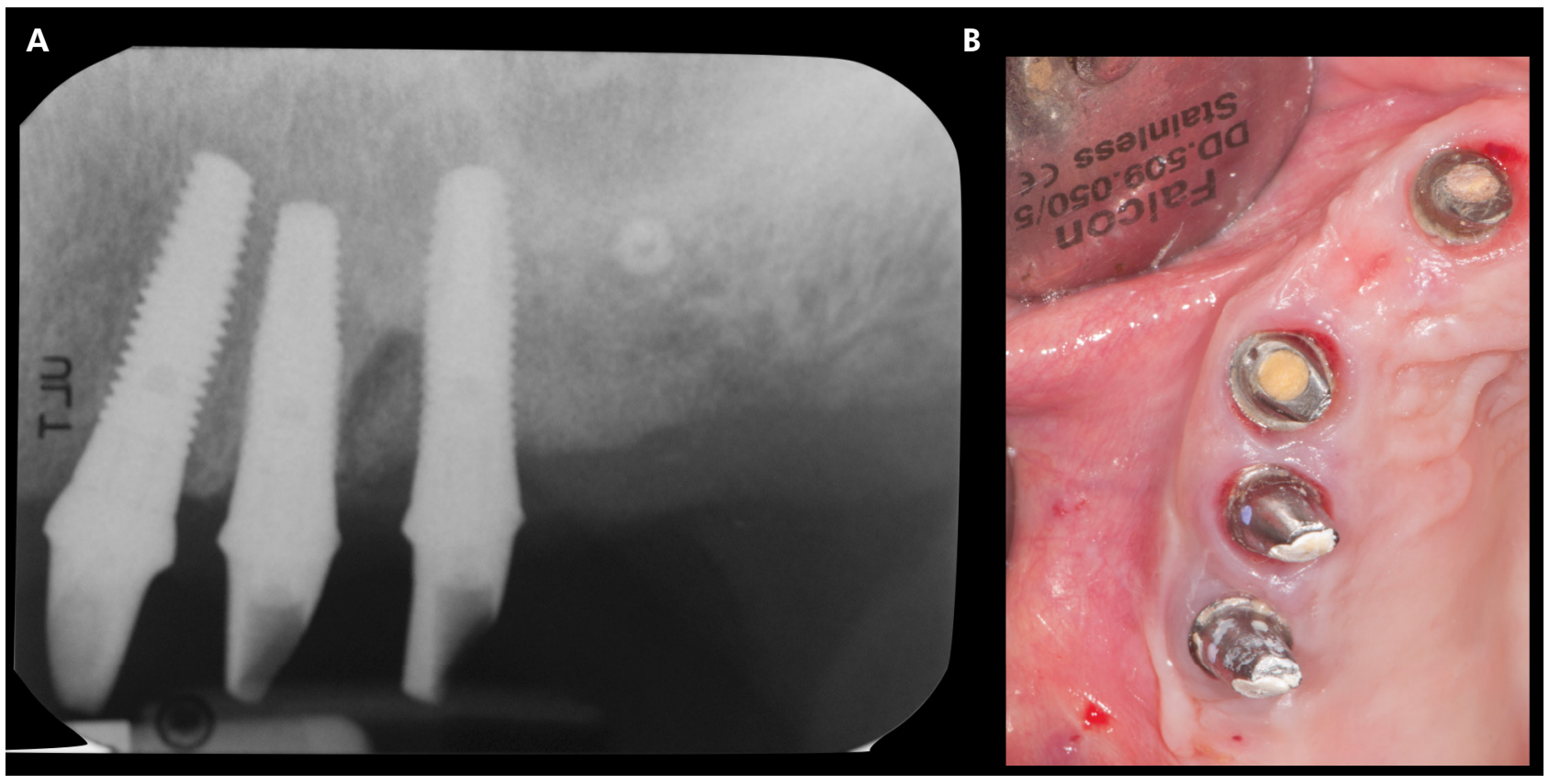

Figure 1. Preoperative radiograph (a) and clinical appearance of the defect (b).

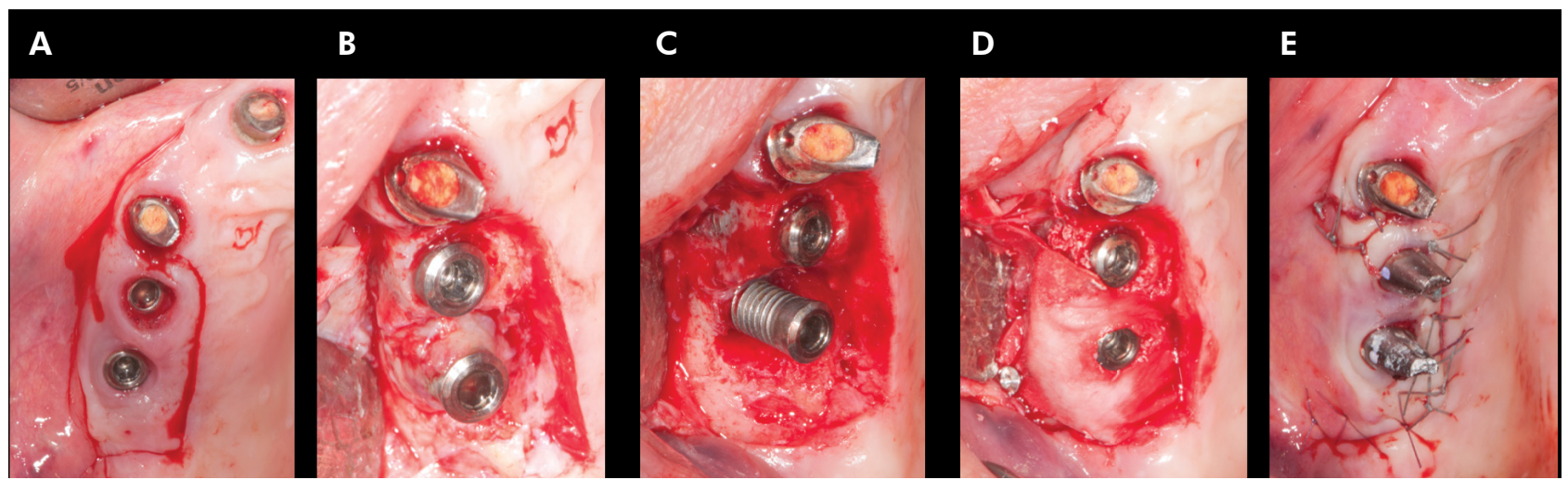

Figure 2. Surgical phases: flap design, intraoperative view of the lesion (b), degranulation of the defect (c), autogenous bone graft + allograft apposition covered by the membrane fixed buccally and palatally using titanium pins (d), primary intention flap suturing (e).

further cleaned/disinfected with a glycin powder spray and an antibiotic paste (Mynocycline). The inside of the implant connection was cleaned using chlorhexidine 0,2\% (GUM Paroex 0.2\%, Sunstar Suisse, Etoy, Switzerland). Bone perforations were performed to increase blood supply and the peri-implant bone defects were filled using autologous bone (collected from neighboring area using scrapers) and an alloplastic bone graft substitute (Genoss, Osteobiol, Turin, Italy) in a 50:50 rate to fill the remaining gap. A membrane
(Evolution, Osteobiol, Turin, Italy) was adapted over the defect and fixed using metallic pins.

Prosthetic abutments and rehabilitations were cleaned according to Canullo et al. ${ }^{7}$ using ultrasounds and an extraoral plasma of argon device (Plasma R, Diener Electronic $\mathrm{GmbH}$, Jettingen, Germany). Flap tensionless soft tissue closure was performed with $5 / 0$ suture. Sutures were removed 2 weeks after the intervention (Figure 2). One to two months thereafter, cleaned prosthetic components were re-inserted after a micro-surgical re-opening. 


\section{Follow-up and maintenance}

Before suture removal, patients were advised to discontinue tooth brushing and to avoid trauma at the site of surgery. After the healing phase, patients were placed on an individually tailored maintenance care program. Motivation, reinforcement of oral hygiene instruction, supragingival instrumentation and antiseptic therapy were performed as needed. Nonsurgical treatment with ultrasound plastic instruments and Erythritol powder were repeated every three months throughout the whole of follow-up period.

\section{Clinical assessment}

All clinical assessments were performed by a single trained clinician using a predefined standard protocol. The following clinical variables were assessed at 6 and 12 months with the aid of a periodontal probe with a millimeter-scale (Hawe Neos Probe 1395, Hawe, UK.):

a. Plaque index $(\mathrm{Pi})$;

b. Bleeding on probing (BoP) considered positive if bleeding was evident within $30 \mathrm{sec}$ after probing, or negative if no bleeding was noticed within 30 sec after probing;

c. Probing depth (PD) measured from the mucosal margin to the bottom of the examined pocket;

d. Mucosal recession (MR) measured from the implant shoulder (IS)/restoration margin to the mucosal margin;

e. Clinical attachment level (CAL) measured from the implant neck to the deepest point of the periimplant pocket;

f. PD, MR, and CAL scores were recorded to the nearest millimeter at six aspects per implant.

\section{Radiographic assessment}

Radiographic changes (bone gain/loss) were evaluated using periapical radiographs obtained at baseline, at 6 and 12 months using paralleling rings to reproduce the exact film position. Peri-implant marginal bone changes were evaluated with a computerized measuring technique applied to digital radiographs.

The distance from the mesial and distal margin of the implant neck to the most coronal point where the bone appeared to be in contact with the implant was measured. Evaluation of the marginal bone level around implants was performed using an image analysis software (Scion Image 4.02 Win, Scion corporation, Frederick, USA) able to compensate radiographic distortion. The software calculated bone remodeling at the mesial and distal aspects of the implants. Mean of both values was used.

\section{Statistical analysis}

The statistical analysis was performed using a commercially available software program (PASW Statistics 20.0; SPSS Inc., Chicago, USA). Mean values and standard deviations were calculated for each variable using the implant as statistical unit. The data rows were examined with the Kolmogorov-Smirnov test and proven to be normally distributed. For the statistical evaluation of the changes over time, the paired t-test was used. The alpha error was set at 0.05 .

\section{Results}

A total of 6 patients and 13 implants was followed until the 12 months follow-up visit. No patient reported any sign of swelling or pain after the surgery. Clinical and radiological data were summarized in Table. Mean PD values were significantly reduced by $3.9 \mathrm{~mm}$ and $3.2 \mathrm{~mm}$ at 6 and 12 months, respectively. The surgical procedure was associated with a pronounced increase in REC and CAL, however, stable peri-implant conditions at 6 and 12 months were reported. Mean $B O P$ values were significantly reduced both at 6 and 12 months. Before treatment, pus was present around 4 implants. At the end of the observation period implants resulted in healthy conditions.

All patients revealed low PI values throughout the entire observation period of 12 months. Radiological analysis demonstrated a complete or semi-complete filling of the defect in all the cases, with a significant bone gain both at 6 and 12 months (Figures 3 and 4).

\section{Discussion}

The present clinical study aimed to analyze a clinical approach based on disinfection of implant connection, disinfection of the implant surface and GBR approach in the treatment of circumferential and semi-circumferential bony defect. 
Table. Clinical parameters (mean \pm SD) at baseline and 6 months and 12 months (13 implants).

\begin{tabular}{lcccc}
\hline Variable & Baseline & 6-month follow-up & 12-month follow-up & p-value * \\
\hline Plaque Index (PI) & $0.36 \pm 0.61$ & $0.06 \pm 0.02$ & $0.0 \pm 0.0$ & 0.003 \\
Bleding on probing (BOP) & $77.1 \pm 11.4 \%$ & $24.09 \pm 12.2 \%$ & $21.1 \pm 17.8 \%$ & 0.000 \\
Probing Depth (PD) & $7.8 \pm 1.6 \mathrm{~mm}$ & $3.1 \pm 1.2 \mathrm{~mm}$ & $3.3 \pm 1.1 \mathrm{~mm}$ & 0.000 \\
Mucosal Recession (MR) & $0.65 \pm 0.51 \mathrm{~mm}$ & $-0.3 \pm 0.2 \mathrm{~mm}$ & $-0.3 \pm 0.2 \mathrm{~mm}$ & 0.002 \\
Clinical Attachment Level (CAL) & $7.8 \pm 1.8 \mathrm{~mm}$ & $3.5 \pm 1.4 \mathrm{~mm}$ & $3.6 \pm 1.7 \mathrm{~mm}$ & 0.003 \\
Rx Bone Loss & $6.1 \pm 0.9 \mathrm{~mm}$ & $1.2 \pm 0.6 \mathrm{~mm}$ & $1.0 \pm 0.4 \mathrm{~mm}$ & 0.0004 \\
\hline
\end{tabular}

Comparison within group (paired t-test).

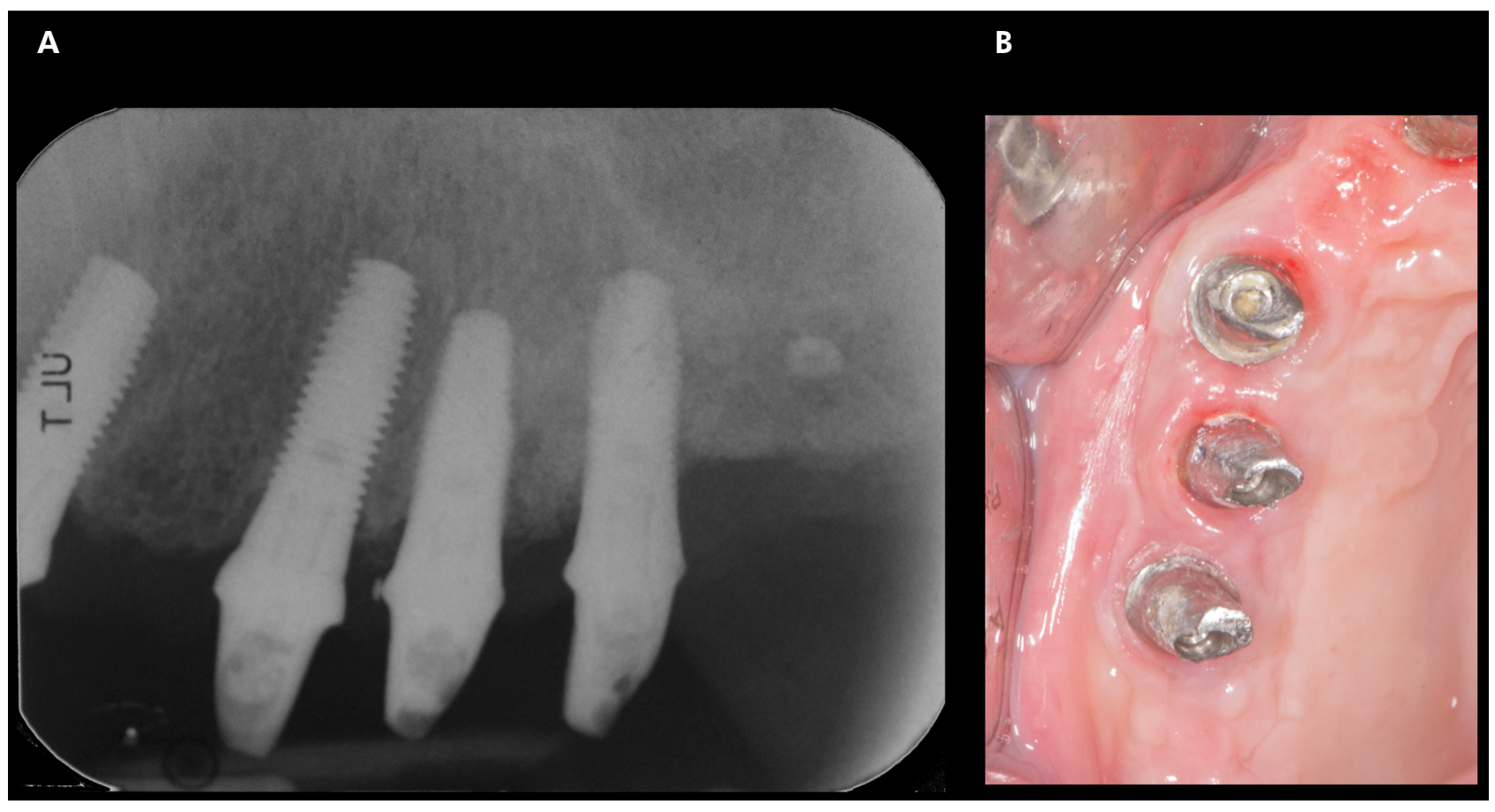

Figure 3. Six-month follow-up radiograph (a) and clinical appearance (b).

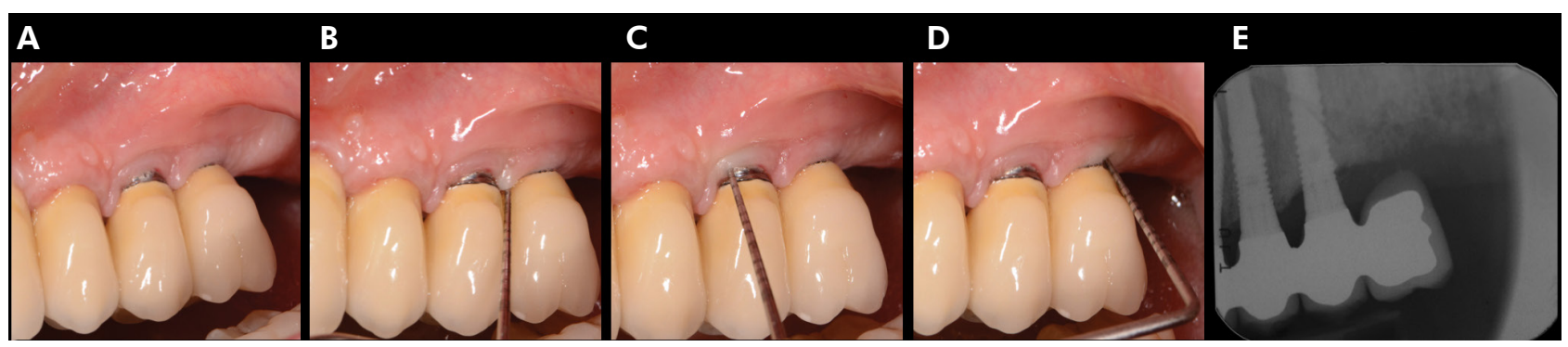

Figure 4. Clinical (a-d) and radiographic (e) view at the 12-month follow-up.

The results of the present research reported a mean PD gain of $4.5 \mathrm{~mm}$, a bone loss reduction of 5.1 $\mathrm{mm}$ and stable peri-implant tissues at the 12-months follow-up visit. GBR approach in the treatment of circumferential and semi-circumferential bony defect seems a viable treatment alternative. 
Nevertheless, the perception among clinicians is that the peri-implantitis treatment is unpredictable and may not lead to success. ${ }^{8}$

Esposito et al. ${ }^{9}$ in a systematic review on the therapy found that in some studies with a follow-up longer than 1 year, recurrence of peri-implantitis was up to $100 \%$ of the treated cases. In contrast, Renvert and coworkers found that stable clinical results could be achieved up to 5 years after initial therapy but highlighting that adequate oral hygiene across this period appeared to be an essential prerequisite. ${ }^{10}$

Another aspect that must be analyzed and that is a major topic of debate, is the reason for marginal peri-implant bone loss. Different causes and etiology have been proposed like adverse occlusal loading effects from hyper-contacts, ${ }^{11,12}$ unfavorable healing, ${ }^{13}$ the effect of position and adaption of the microgap, ${ }^{14}$ plaque accumulation, implant malposition, ${ }^{14}$ cement excess, ${ }^{15}$ smoking or a multi-factorial etiology. ${ }^{14}$

Theoretically, etiological treatment focusing on the pathophysiology of the disease should be the objective of the management of the pathology. The unclear peri-implantitis etiology made questionable every possible treatment and the long-term result of it.

The approach presented in the present research is a combination of "common sense" procedures applied to eliminate or reduce the possible risk factors.

Great attention was paid in the decontamination of both the implant surface and connection. Plasma of argon has demonstrated its capability to disinfected the implant surface, with potential to promote osteoblast attachment and spreading. ${ }^{16}$

The implant surface was carefully debrided mechanically and rinsed with a powder spray.
Air-powder abrasives were studied in a number of articles which showed that the use of amino acid glycine powder can be effective in removal of bacterial biofilm without altering the morphological characteristics of implant surface. ${ }^{17,18,19}$ Further, air-abrasive powder has the advantage of preserving the surface characteristics of titanium without creating roughness and alterations that can become a bacterial niche. ${ }^{17,20}$

Finally, an antibiotic paste (mynociclin) was used. Minocycline seems to provide significant benefits in terms of clinical parameters and radiographic bone fill, with a higher treatment success rate in the short healing period. ${ }^{21}$

Ultimately, supportive care of patients was reinforced. As stated by Roccuzzo et al., therapy of peri-implantitis followed by regular supportive care resulted in high patient- and implant-level survival in the medium to long term. ${ }^{8}$

Some limits of the present research must be acknowledged, in particular the low number of treated patients and the short follow-up period that do not consent to generalize the results. RCT studies comparing GBR treatment to noninvasive debridement in peri-implantitis cases are needed in order to provide evidence for an additional benefit of the use of bone graft substitutes.

\section{Conclusions}

Within the limitation of the present preliminary study, the proposed technique might represent a promising result for the treatment of circumferential and semi-circumferential bone defects around implants affected by peri-implantitis.

\section{References}

1. Jung RE, Pietursson BE, Glauser R, Zembic A, Zwahlen M, Lang NP. A systematic review of the 5-year survival and complication rates of implant-supported single crowns. Clin Oral Implants Res. 2008 Feb;19(2):119-30. https://doi.org/10.1111/j.1600-0501.2007.01453.x

2. Derks J, Tomasi C. Peri-implant health and disease: a systematic review of current epidemiology. J Clin Periodontol. 2015 Apr; 42 Suppl 16:S158-71. https://doi.org/10.1111/icpe.12334

3. Pesce P, Menini M, Tealdo T, Bevilacqua M, Pera F, Pera P. Peri-implantitis: a systematic review of recently published papers. Int J Prosthodont. 2014 Jan-Feb;27(1):15-25. https://doi.org/10.11607/ijp.3785

4. Madi M, Htet M, Zakaria O, Alagl A, Kasugai S. Re-osseointegration of dental implants after periimplantitis treatments: a systematic review. Implant Dent. 2018 Feb;27(1):101-10. https://doi.org/10.1097/ID.0000000000000712 
5. Sahrmann P, Attin T, Schmidlin PR. Regenerative treatment of peri-implantitis using bone substitutes and membrane: a systematic review. Clin Implant Dent Relat Res. 2011 Mar;13(1):46-57. https://doi.org/10.1111/j.1708-8208.2009.00183.x

6. Schwarz F, Sahm N, Schwarz K, Becker J. Impact of defect configuration on the clinical outcome following surgical regenerative therapy of peri-implantitis. J Clin Periodontol. 2010 May;37(5):449-55. https://doi.org/10.1111/j.1600-051X.2010.01540.x

7. Canullo L, Penarrocha D, Micarelli C, Massidda O, Bazzoli M. Hard tissue response to argon plasma cleaning/sterilisation of customised titanium abutments versus 5 -second steam cleaning: results of a 2-year post-loading follow-up from an explanatory randomised controlled trial in periodontally healthy patients. Eur J Oral Implantol. 2013;6(3):251-60.

8. Roccuzzo M, Layton DM, Roccuzzo A, Heitz-Mayfield LJ. Clinical outcomes of peri-implantitis treatment and supportive care: a systematic review. Clin Oral Implants Res. 2018 Oct;29 Suppl 16:331-50. https://doi.org/10.1111/clr.13287

9. Esposito M, Grusovin MG, Worthington HV. Interventions for replacing missing teeth: treatment of peri-implantitis. Cochrane Database Syst Rev. 2012 Jan;1:CD004970. https://doi.org/10.1002/14651858.CD004970.pub5

10. Renvert S, Polyzois I, Persson GR. Treatment modalities for peri-implant mucositis and peri-implantitis. Am J Dent. 2013 Dec;26(6):313-8.

11. Hälg GA, Schmid J, Hämmerle CH. Bone level changes at implants supporting crowns or fixed partial dentures with or without cantilevers. Clin Oral Implants Res. 2008 Oct;19(10):983-90. https://doi.org/10.1111/i.1600-0501.2008.01556.x

12. Oh TJ, Yoon J, Misch CE, Wang HL. The causes of early implant bone loss: myth or science? J Periodontol. 2002 Mar;73(3):322-33. https://doi.org/10.1902/jop.2002.73.3.322

13. King GN, Hermann JS, Schoolfield JD, Buser D, Cochran DL. Influence of the size of the microgap on crestal bone levels in non-submerged dental implants: a radiographic study in the canine mandible. J Periodontol. 2002 Oct;73(10):1111-7. https://doi.org/10.1902/jop.2002.73.10.1111

14. Canullo L, Schlee M, Wagner W, Covani U. International Brainstorming Meeting on Etiologic and Risk Factors of Periimplantitis, Montegrotto (Padua, Italy), August 2014. Int J Oral Maxillofac Implants. 2015 Sep-Oct;30(5):1093-104. https://doi.org/10.11607/jomi.4386

15. Pesce P, Canullo L, Grusovin MG, Bruyn H, Cosyn J, Pera P. Systematic review of some prosthetic risk factors for periimplantitis. J Prosthet Dent. 2015 Sep;114(3):346-50. https://doi.org/10.1016/i.prosdent.2015.04.002

16. Canullo L, Genova T, Wang HL, Carossa S, Mussano F. Plasma of Argon increases cell attachment and bacterial decontamination on different implant surfaces. Int J Oral Maxillofac Implants. 2017 Nov/Dec;32(6):1315-23. https://doi.org/10.11607/jomi.5777

17. Taschieri S, Weinstein R, Del Fabbro M, Corbella S. Erythritol-enriched air-polishing powder for the surgical treatment of peri-implantitis. ScientificWorldJournal. 2015;2015:802310. https://doi.org/10.1155/2015/802310

18. Petersilka G, Faggion CM Jr, Stratmann U, Gerss J, Ehmke B, Haeberlein I, et al. Effect of glycine powder air-polishing on the gingiva. J Clin Periodontol. 2008 Apr;35(4):324-32. https://doi.org/10.1111/j.1600-051X.2007.01195.x

19. Flemmig TF, Arushanov D, Daubert D, Rothen M, Mueller G, Leroux BG. Randomized controlled trial assessing efficacy and safety of glycine powder air polishing in moderate-to-deep periodontal pockets. J Periodontol. 2012 Apr;83(4):444-52. https://doi.org/10.1902/jop.2011.110367

20. Lin HY, Liu Y, Wismeijer D, Crielaard W, Deng DM. Effects of oral implant surface roughness on bacterial biofilm formation and treatment efficacy. Int J Oral Maxillofac Implants. 2013 Sep-Oct;28(5):1226-31. https://doi.org/10.11607/jomi.3099

21. Cha JK, Lee JS, Kim CS. Surgical therapy of peri-implantitis with local minocycline: a 6-month randomized controlled clinical trial. J Dent Res. 2019 Mar;98(3):288-95. https://doi.org/10.1177/0022034518818479 